\title{
ÍNDICE DE ADIPOSIDADE VISCERAL COMO PREDITOR DE RISCO CARDIOMETABÓLICO EM CRIANÇAS E ADOLESCENTES
}

\author{
VISCERAL ADIPOSITY INDEX AS A PREDICTOR OF CARDIOMETABOLIC RISK IN CHILDREN \\ ANDADOLESCENTS \\ ÍNDICE DEADIPOSIDAD VISCERAL COMO PREDICTOR DEL RIESGO CARDIOMETABÓLICO \\ EN NINNOS Y ADOLESCENTES
}

Artigo Original

Original ARTICLE

Artículo Original

\begin{abstract}
Ronano Pereira Oliveira ${ }^{1,2}$ (Profissional de Educação Física) Jane Maria Remor' (Profissional de Educação Física) Adriano Ruy Matsuo (Profissional de Educação Física) Rafaela Pilegi Dada ${ }^{1}$ (Profissional de Educação Física) Anselmo Alexandre Mendes (Profissional de Educação Física) Tamires Leal Cordeiro dos Santos ${ }^{1}$ (Profissional de Educação Física) João Carlos Locateli' (Graduando em Educação Física) Caio Machado de Oliveira Terra' (Profissional de Educação Física) Samara Pereira Brito Lazarin' (Profissional de Educação Física) Nelson Nardo Junior (Profissional de Educação Física)

1. Universidade Estadual de Maringá. Maringá, PR, Brasil. 2. Instituto Federal do Tocantins. Araguatins, TO, Brasil.
\end{abstract}

\section{Correspondência:}

Nelson Nardo Júnior Universidade Estadual de Maringá, Departamento de Educação Física. Av. Colombo, 5.790, Zona 07, Maringá, PR, Brasil. 87020-900. nnjunior@uem.br; ronano@ifto.edu.br

\section{RESUMO}

Introdução: O risco cardiometabólico está associado a diversos fatores, entre os quais, o estado nutricional tem papel destacado. Objetivos: Este estudo verificou a influência do estado nutricional sobre o Índice de Adiposidade Visceral (VAl, do inglês) e seus componentes em crianças e adolescentes. Métodos: Estudo descritivo-associativo, de caráter transversal, realizado com 290 sujeitos de 10 a 18 anos, de ambos os sexos. Foram avaliados estatura, massa corporal, IMC, HDL-c, TG e VAI. Realizaram-se os testes Shapiro-Wilk, teste $t$ de Student independente, U de Mann-Whitney, ANOVA One-Way, Kruskal-Wallis, Post Hoc de Bonferroni, Correlação de Pearson e cálculo da razão de chances (odds ratio). A significância adotada foi de $p<0,05$. Resultados: Os testes demonstraram haver diferenças significativas entre os sexos para a idade, HDL-c e VAl. Nas variáveis HDL-C, TG e VAl foram constatadas diferenças significativas entre os adolescentes eutróficos e as demais classificações do estado nutricional. Verificou-se também uma piora das variáveis utilizadas para o cálculo do VAl para os adolescentes com classificação do estado nutricional alterado (não eutróficos), indicando, portanto, maior risco cardiometabólico associado ao excesso de peso. Verificou-se forte correlação entre o VAl e o TG $(0,865)$ e correlação moderada negativa entre VAl e HDL-c $(-0,405)$. Após o odds ratio, constatou-se que os participantes com excesso de peso e CC alterada, respectivamente, apresentaram 2,3 e 1,5 vezes mais chance de risco cardiometabólico (VAl alterado). Conclusões: O estado nutricional é capaz de influenciar as variáveis de risco cardiometabólico, VAl, CC, TG e HDL-c em crianças e adolescentes. Nesta população, independente do sexo, à medida que o valor do IMC se eleva, há progressão de alterações metabólicas. Em complemento, o VAl apresentou uma correlação significativa com TG, HDL-c, IMC e CC das crianças e adolescentes do estudo, podendo assim ser visto como alternativa de predição do risco cardiometabólico.

Descritores: obesidade; atividade motora; educação física e treinamento.

\section{ABSTRACT}

Introduction: The cardiometabolic risk is associated with several factors, among which nutritional status plays a prominent role. Objectives: This study verified the influence of nutritional status on the Visceral Adiposity Index (VAI) and its components in children and adolescents. Methods: Descriptive, associative, cross-sectional study performed with a sample of 290 individuals from 10 to 18 years of age of both sexes. We assessed height, body mass, BMI, HDL-C, $T G$, and VAI. The following tests were used: Shapiro-Wilk, independent Student t test, Mann-Whitney U-test, ANOVA One-Way, Kruskal-Wallis, Bonferroni post hoc test, Pearson Correlation and odds ratio. The significance level was established at $p<0.05$. Results: The tests showed significant differences between the sexes for age, HDL-c, and VAl. Concerning the variables HDL-C, TG and VAl, significant differences were observed among the eutrophic adolescents and the other classifications of nutritional status. There was also a worsening of the variables used to calculate VAl for adolescents with altered nutritional status (non-eutrophic), indicating a higher cardiometabolic risk associated with overweight There was a strong correlation between VAl and TG (0.865) and moderate negative correlation between VAl and HDL-C (-0.405). After the odds ratio calculation, it was found that participants with overweight and altered WC, respectively presented 2.3 and 1.5 times more chance of cardiometabolic risk (altered VAl). Conclusions: The nutritional status is capable of influence the variables of cardiometabolic VAl, WC, TG, and HDL-cin children and adolescents. In this population, regardless of sex, as the BMI increases, there is a progression of metabolic changes. In addition, VAl index showed a significant correlation with TG, HDL-C, BMI, and WC of the children and adolescents in the study, which could be seen as an alternative to predict metabolic risk.

Keywords: obesity; motor activity; physical education and training.

\section{RESUMEN}

Introducción: El riesgo cardiometabólico se asocia con varios factores, incluyendo el estado nutricional, que tiene un papel importante. Objetivos: Este estudio examinó la influencia del estado nutricional sobre el Índice de Adiposidad Visceral (VAl, del inglés) y sus componentes en niños y adolescentes. Métodos: Estudio descriptivo-asociativo, de carácter transversal, realizado con 290 sujetos de 10 a 18 años, de ambos sexos. Se evaluó la altura, la masa corporal, IMC, HDL-C, TG y VAI. Fueron realizadas las pruebas Shapiro-Wilk, prueba t de Student independiente, prueba U de 
Mann-Whitney, ANOVA One-Way, Kruskal-Wallis, prueba post hoc de Bonferroni, Correlación de Pearson y cálculo de la razón de posibilidades (odds ratio). La significación adoptada fue de $p<0,05$. Resultados: Las pruebas demostraron diferencias significativas entre los sexos para la edad, HDL-cy VAI. En las variables HDL-c, TG y VAl se constataron diferencias significativas entre los adolescentes eutróficos y las otras clasificaciones del estado nutricional. También hubo un empeoramiento de las variables utilizadas para el cálculo del VAl para los adolescentes con clasificación de estado nutricional alterado (no eutróficos), lo que indica un mayor riesgo cardiometabólico asociado con el exceso de peso. Hubo una fuerte correlación entre VAl e TG $(0,865)$ y correlación negativa moderada entre VAly HDL-c $(-0,405)$. Después del cálculo de la razón de posibilidades se encontró que los participantes con sobrepeso y CC alterada, respectivamente, presentaron 2,3 y 1,5 veces más probabilidades de riesgo cardiometabólico (VAl alterado). Conclusiones: El estado nutricional puede influir en las variables de riesgo cardiometabólico, VAl, CC, TG y HDL-c en niños y adolescentes. En esta población, independientemente del sexo, cuando el valor del IMC se eleva, hay progresión de los cambios metabólicos. Además, el VAl mostró una correlación significativa con TG, HDL-C, IMC y CC de los niños y adolescentes del estudio y, por lo tanto, se puede considerar como un predictor alternativo de riesgos cardiometabólicos.

Descriptores: obesidad; actividad motora; educación y entrenamiento físico.

\section{INTRODUÇÃO}

A prevalência de excesso de peso alcançou proporções alarmantes por todo o mundo. A obesidade se destaca entre as doenças crônicas não transmissíveis (DCNTs) por ser um fator de risco para outras DCNTs como a hipertensão arterial e o diabetes mellitus tipo 2, tornando-se um desafio para a saúde pública no Brasil, em função das causas multifatoriais e complexidade de tratamento 1,2. Em consonância com esse cenário encontram-se crianças e adolescentes brasileiros estudados em levantamentos nacionais e internacionais ${ }^{3,4}$.

Dentre os vários indicadores antropométricos, o Índice de Massa Corporal (IMC) é o mais utilizado para estimar a prevalência de obesidade em uma população e os riscos associados ao excesso de peso, embora não leve em conta a ampla variação na quantidade e distribuição da gordura corporal ${ }^{5,6}$. Além disso, esse parâmetro apresenta resultados controversos em diversas populações, de modo que carece de complementação como indicador de risco cardiometabólico.

Nessa direção, Amato et al. ${ }^{7}$ defendem a necessidade de um método diagnóstico ou equação de predição de baixo custo que estime a gordura visceral e gordura subcutânea separadamente, uma vez que, a gordura visceral está diretamente relacionada com o desencadeamento de distúrbios metabólicos ${ }^{8}$ e os métodos de imagem por ressonância magnética e tomografia computadorizada considerados padrão-ouro pela comunidade científica são muitas vezes inacessíveis a grande parcela da população devido a seu alto custo9 .

Com base nisso, Amato et al. ${ }^{7}$ criaram e validaram um novo preditor da distribuição e da função da gordura visceral associado ao risco cardiometabólico, denominado Visceral Adiposity Index (VAI). A partir de então, diversos estudos têm analisado a utilidade prática desse índice, entre os quais, se inclui uma pesquisa realizada com adultos árabes na qual o VAl foi o melhor preditor de diabetes mellitus tipo 2 e Síndrome Metabólica (SM) em comparação a outros índices como o IMC e o Body Adipositylndex (BAI) ${ }^{10}$. Resultados semelhantes também foram encontrados em chineses adultos ${ }^{11}$.

Além disso, foi constatado que o VAI pode ser um preditor independente de hipertensão arterial, bem como de pré-hipertensão arterial em indivíduos adultos de ambos os sexos ${ }^{12}$. No Brasil, Schuster et al. ${ }^{13}$ realizaram um estudo com adultos saudáveis no qual o VAl apresentou associação com componentes da SM em homens e mulheres, como obesidade abdominal, hiper trigliceridemia e baixos níveis de HDL colesterol (HDL-c). Zen ${ }^{14}$ investigou a associação do VAl com a doença arterial coronariana em adultos concluindo que o VAl foi significativamente associado à incidência de eventos cardiovasculares adversos maiores.
Em indivíduos adultos obesos de ambos os sexos, VAl apresenta um valor elevado, independentemente de serem metabolicamente saudáveis ou não, quando comparados a indivíduos sobrepesados ou com peso normal ${ }^{15}$. Desta forma, o estado nutricional parece exercer influência sobre o VAl, o que sugere um aumento para o risco cardiometabólico de acordo com o valor do IMC.

A utilidade do VAI como um índice diagnóstico de risco cardiometabólico na população adulta está sendo adequadamente documentada. Entretanto, não se tem conhecimento sobre a utilidade desse índice diagnóstico na população infanto-juvenil brasileira. Além disso, até onde temos conhecimento não há estudos publicados que avaliem o efeito do estado nutricional de crianças e adolescentes brasileiros sobre o VAl, de modo a verificar a viabilidade de sua utilização como marcador de risco cardiometábolico para esta população. Assim, o objetivo deste estudo foi verificar a influência do estado nutricional sobre o VAl, a circunferência da cintura (CC), os níveis de HDL-c e de triglicerídeos (TG) em crianças e adolescentes conforme o sexo.

\section{MATERIAIS E MÉTODOS}

Este é um estudo descritivo-associativo, de caráter transversal, realizado com 290 crianças e adolescentes, na faixa etária entre 10 e 18 anos, de ambos os sexos, participantes de dois projetos desenvolvidos pelo Núcleo de Estudos Multiprofissional da Obesidade (NEMO - UEM).

Um dos projetos tinha como objetivo avaliar os fatores de risco cardiometabólicos em adolescentes com sobrepeso e obesidade de Maringá-PR, e verificar os efeitos de um Programa Multiprofissional de Tratamento da Obesidade (PMTO) nessa população. O outro projeto tinha como objetivo investigar a associação entre o estado nutricional e os fatores de risco cardiometabólicos em escolares de uma cidade de pequeno porte do noroeste do Paraná.

Ambos os projetos foram aprovados pelo Comitê Permanente de Ética em Pesquisa com Seres Humanos da Universidade Estadual de Maringá. O primeiro aprovado sob os pareceres no 463/2009 e n 915.526/2014 e o segundo sob $n^{\circ} 1.313 .449 / 2015$. Ambos os estudos atenderam os regulamentos exigidos pela Resolução do Conselho Nacional de Saúde no 466/2012 e a Declaração de Helsinque (1964). Este estudo está cadastrado no Registro Brasileiro de Ensaios Clínicos (ReBEC) sob no RBR-45ywtg (www.ensaiosclinicos.gov.br/rg/RBR-45ywtg/).

Os participantes, 59\% do sexo feminino, foram recrutados através de divulgação em escolas e pela mídia local. Após a divulgação dos projetos foram realizadas reuniões com os responsáveis e interessados para apresentação dos objetivos e dos protocolos de avaliação e intervenção, aos quais os mesmos seriam submetidos. Participaram do estudo 
aqueles que junto com seus responsáveis concordaram e assinaram o Termo de Consentimento Livre e Esclarecido.

A estatura dos sujeitos foi medida por meio de um estadiômetro (Sanny, modelo ES 2030, São Bernardo do Campo/SP, Brasil) com precisão de 0,1 cm com campo de uso de $0,80 \mathrm{~m}$ a 2,20m, seguindo os procedimentos recomendados por Lohman, Roche e Martorell ${ }^{16}$. A massa corporal foi medida com aparelho de bioimpedância octapolar multifrequencial (Biospace, modelo 520 - InBody Composition Analysers, Coréia). Para estas medidas os participantesforam orientados a seguir as recomendações de comparecer ao laboratório no período vespertino, após jejum de 2 horas e antes do início da avaliação, retirar seus calçados e objetos metálicos que estivessem portando. A partir destas medidas foi calculado o IMC e classificado de acordo com os valores propostos por Cole eLobstein ${ }^{17}$.

A CC foi medida com uma trenaantropométrica não extensiva (Sanny, modelo SN-4010, São Bernardo do Campo/SP, Brasil) com capacidade de medir até 2 metros e precisão de 0,1 cm, conforme Lohman, Roche e Martorell ${ }^{16}$.

As coletas de sangue para a mensuração das variáveis lipídicas foram realizadas por especialistas de um laboratório comercial de referência da cidade de Maringá com certificação ISO 9002 (International Organization for Standardization), utilizando-se kits da marca Siemens ${ }^{\circledR}$ (Frimley, Camberley, Grã-Bretanha), após $10 \pm 2$ horas de jejum, no período da manhã entre 7 e 10 horas. Foram mensuradasas frações HDL-c e a concentração de TG, o sangue venoso foi armazenado em tubos tipo Vacutainer ${ }^{\circledR}$ com gel separador, sendo as análises conduzidas por meio do soro. As amostras foram centrifugadas a 3.600 rpm durante 11 minutos em temperatura ambiente e foram analisadas pelo aparelho Siemens Advia 1800 Chemistry Analyser $^{\circledR}$.

O cálculo do VAI foi feito a partir da fórmula proposta por Amato et al. ${ }^{18}$. A fórmula é composta por duas medidas antropométricas (IMC em Kg/m² e CC em cm) e dois parâmetros metabólicos (HDL-c e $\mathrm{TG}$ em $\mathrm{mmol} / \mathrm{L})^{7,19}$. Para o sexo masculino VAI $=(\mathrm{CC} / 39,68+(1,88 \times \mathrm{IMC}))$ $\times(T G / 1,03) \times(1,31 / \mathrm{HDL})$ e para o sexo feminino $\mathrm{VAI}=(\mathrm{CC} / 36,58+(1,89 \times$ IMC) $) \times(T G / 0,81) \times(1,52 / H D L)$.

Após a realização das coletas e tabulação dos dados foi utilizado o teste de Shapiro-Wilk para verificar a normalidade dos dados. Assim, os dados normais foram apresentados em média e desvio-padrão e os dados não-normais apresentados em mediana e intervalo interquartil. Posteriormente, para análise inferencial dos dados, foram utilizados os testes $t$-Student para amostras independentes e $U$ de Mann-Whitney para comparação entre sexos. Os testes ANOVA One-Way, Kruskal-Wallis e o Post Hoc de Bonferroni foram utilizados para comparação entre os estados nutricionais. Para a análise de correlação das variáveis foi utilizado o teste de correlação de Pearson. Foi ainda calculada a razão de chance (Odds Ratio) para o VAl e seus determinantes antropométricos (IMC e CC). A significância adotada foi de $p<0,05$. As análises foram processadas utilizando-se o pacote estatístico da IBM, Software SPSS versão 20.0.

\section{RESULTADOS}

A Tabela 1 apresenta os resultados das características gerais dos participantes da pesquisa, estratificados por sexo, em medidas de tendência central e dispersão. Os testes demonstraram haver diferenças significativas entre os sexos para as variáveis: idade, HDL-c e VAl, com valores mais elevados para o sexo feminino, e massa corporal, estatura e CC, para o sexo masculino.

A Tabela 2 apresenta a comparação do VAl, de acordo com o estado nutricional dos participantes da pesquisa. Observou-se diferença significativa na idade entre os participantes eutróficos em relação aos com classificação de obesidade grave. Em relação a obesidade abdominal, com base na medida de CC, foi constatada diferença significativa entre todos os estados nutricionais. Nas variáveis bioquímicas HDL-c e TG, e também no indicador de risco VAl, foram constatadas diferenças estatisticamente significativas entre os adolescentes eutróficos e as demais classificações do estado nutricional.

Verificou-se também uma piora das variáveis utilizadas para o cálculo do VAl para os adolescentes com classificação do estado nutricional alterado (não eutróficos), indicando, portanto, maior risco cardiometabólico associado ao excesso de peso.

A Tabela 3 apresenta a comparação das variáveis: CC, HDL-C, TG e VAl entre os estados nutricionais, estratificados por sexo. Em ambos os sexos foram encontradas diferenças significativas para: CC entre todos os estados nutricionais; TG e VAl entre eutróficos e os demais estados nutricionais. Para o sexo masculino o HDL-c apresentou diferenças significativas entre eutróficos e obesos; eutróficos e obesos graves e para o sexo feminino apenas entre eutróficos e sobrepesados.

A Figura 1 apresenta os valores de correlação entre o VAl e os demais parâmetros analisados. Verificou-se haver forte correlação entre o VAl e o TG $(0,865)$ e correlação moderada negativa entre VAl e HDL-c $(-0,405)$

Tabela 1. Características gerais dos participantes da pesquisa estratificados por sexo.

\begin{tabular}{c|c|c|c}
\hline Variável & $\begin{array}{c}\text { Masculino } \\
\text { (n= 119) }\end{array}$ & $\begin{array}{c}\text { Feminino } \\
\text { (n= 171) }\end{array}$ & $\mathbf{P}$ \\
\hline Idade & $15(3)$ & $16(1)$ & $0,006^{*}$ \\
\hline Massa corporal $(\mathrm{Kg})$ & $87,72 \pm 26,05$ & $75,24 \pm 18,00$ & $<0,001^{*}$ \\
\hline Estatura $(\mathrm{m})$ & $1,73(0,13)$ & $1,61(0,09)$ & $<0,001^{*}$ \\
\hline IMC $\left(\mathrm{kg} / \mathrm{m}^{2}\right)$ & $28,40(9,70)$ & $28,20(6,83)$ & 0,483 \\
\hline $\mathrm{CC}(\mathrm{cm})$ & $91,15 \pm 14,33$ & $82,25 \pm 11,94$ & $<0,001^{*}$ \\
\hline HDL-c $(\mathrm{mg} / \mathrm{dL})$ & $42,30(10,20)$ & $48,70(15,90)$ & $<0,001^{*}$ \\
\hline $\mathrm{TG}(\mathrm{mg} / \mathrm{dL})$ & $76,90(63,60)$ & $78,00(58,00)$ & 0,701 \\
\hline HDL-c $(\mathrm{mmol})$ & $1,10(0,26)$ & $1,26(0,42)$ & $<0,001^{*}$ \\
\hline $\mathrm{TG}(\mathrm{mmol})$ & $0,87(0,72)$ & $0,88(0,66)$ & 0,700 \\
\hline VAl & $0,963(0,941)$ & $1,236(1,055)$ & $0,005^{*}$ \\
\hline
\end{tabular}

*p $\leq 0,05 \cdot \mathrm{kg}$ quilograma $\mathrm{m}$. metros: IMC. indice de massa corporal. $\mathrm{kg} / \mathrm{m}^{2}$. quilograma por metro quadrado: $C C$. circunferência de cintura; $\mathrm{Cm}$ : centímetros; HDL-c: colesterol lipoproteína de alta densidade; $\mathrm{mg} / \mathrm{dL}$ : miligrama por decilitro; mmol: milimol por litro; TG: triglicerídeos; VAl: visceral adiposity índex

Tabela 2. Comparação da amostra estratificada de acordo com o estado nutricional e fatores relacionados ao Visceral Adipositylndex (VAl).

\begin{tabular}{|c|c|c|c|c|}
\hline \multicolumn{5}{|c|}{ Classificação Nutricional } \\
\hline Variável & $\begin{array}{c}\text { Eutrofia } \\
(n=67-23,10 \%)\end{array}$ & $\begin{array}{c}\text { Sobrepeso } \\
(n=109-37,59 \%)\end{array}$ & $\begin{array}{c}\text { Obesidade } \\
(n=63-21,72 \%)\end{array}$ & $\begin{array}{l}\text { Obesidade grave } \\
(n=51-17,59 \%)\end{array}$ \\
\hline Idade (anos) & $15(3)^{c}$ & $15(3)$ & $15(2)$ & $16(2)^{c}$ \\
\hline $\mathrm{IMC}\left(\mathrm{Kg} / \mathrm{m}^{2}\right)$ & $21,20(4,10)^{a, b, c}$ & $27,50(3,23)^{a, d, e}$ & $31,00(2,70)^{b, d, f f}$ & $37,90(4,50)^{c, e, f}$ \\
\hline $\mathrm{CC}(\mathrm{cm})$ & $70,65 \pm 7,17^{a, b, c}$ & $84,82 \pm 8,37^{a, d, e}$ & $90,53 \pm 7,28^{b, d, f}$ & $102,52 \pm 13,17^{c, e, f}$ \\
\hline $\mathrm{HDL}-\mathrm{c}(\mathrm{mg} / \mathrm{dL})$ & $51,9(15,80)^{a, b, c}$ & $44,9(14,75)^{a}$ & $43,3(13)^{b}$ & $43(12,30)^{c}$ \\
\hline $\mathrm{TG}(\mathrm{mg} / \mathrm{dL})$ & $58(46,20)^{a, b, c}$ & $79(76,50)^{\text {a }}$ & $85(53)^{b}$ & $98(56)^{c}$ \\
\hline $\mathrm{HDL}-\mathrm{c}(\mathrm{mmol})$ & $1,34(0,41)^{a, b, c}$ & $1,16(0,38)^{\mathrm{a}}$ & $1,12(0,34)^{b}$ & $1,11(0,56)^{c}$ \\
\hline TG (mmol) & $0,65(0,52)^{a, b, c}$ & $0,89(0,86)^{\text {a }}$ & $0,961(0,60)^{b}$ & $1,10(0,63)^{c}$ \\
\hline VAl & $0,659(0,797)^{a, b, c}$ & $1,176(1,057)^{\mathrm{a}}$ & $1,326(1,200)^{b}$ & $1,358(0,812)^{c}$ \\
\hline
\end{tabular}

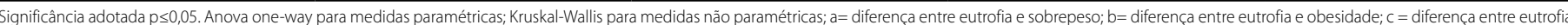
e obesidade grave; $d=$ diferença entre sobrepeso e obesidade; $\mathrm{e}=$ diferença entre sobrepeso e obesidade grave; $\mathrm{f}=$ diferença entre obesidade e obesidade grave. 
sendo que essa foi aqui apresentada de forma invertida, apenas para fins de comparação e melhor ilustração da força de associação em relação aos demais parâmetros, todas as demais correlações são positivas.

A Tabela 4 apresenta as prevalências de alteração das variáveis de estudo. Em ambos os sexos o IMC apresentou altas prevalências de alteração. Os participantes do sexo masculino apresentaram maior prevalência de alteração para a CC e o HDL-c e os do sexo feminino para o TG e para o VAl.

Após o cálculo da razão de chance (Odds Ratio) entre Estado Nutricional vs VAl e Obesidade Abdominal vs VAl, constatou-se que os participantes com excesso de peso apresentaram 2,3 vezes mais chance de terem diagnóstico de risco cardiometabólico (VAI alterado) que os eutróficos. Enquanto que os participantes com circunferência de cintura alterada apresentaram 1,5 vezes mais chance de terem diagnóstico de risco cardiometabólico (VAl alterado) que os com CC Normal.

Tabela 3. Comparação das variáveis: CC, HDL-c, TG e Visceral Adiposity Index (VAl) entre os estados nutricionais, estratificados por sexo.

\begin{tabular}{|c|c|c|c|c|}
\hline \multicolumn{5}{|c|}{ Masculino } \\
\hline Variáveis & Eutrófico & Sobrepeso & Obesidade & $\begin{array}{l}\text { Obesidade } \\
\text { grave }\end{array}$ \\
\hline & $(n=26)$ & $(n=42)$ & $(n=23)$ & $(n=28)$ \\
\hline Idade (anos) & $15(3)$ & $15(3)$ & $15(3)$ & $16(2)$ \\
\hline $\mathrm{CC}(\mathrm{cm})$ & $73,48 \pm 7,60^{\mathrm{a}, \mathrm{b}, \mathrm{c}}$ & $88,71 \pm 8,70^{\mathrm{a}, \mathrm{d}, \mathrm{e}}$ & $95,23 \pm 6,66^{\mathrm{b}, \mathrm{d}, \mathrm{f}}$ & $107,85 \pm 8,94$ ce,,$f$ \\
\hline $\mathrm{HDL}-\mathrm{c}(\mathrm{mg})$ & $48,1(10,63)^{b, c}$ & $42,55(10,13)$ & $39,7(10,40)^{b}$ & $39,9(12)^{c}$ \\
\hline $\mathrm{TG}(\mathrm{mg})$ & $49,2(48)^{a, b, c}$ & $76,85(87,63)^{\mathrm{a}}$ & $88,00(52,00)^{b}$ & $98,00(49,13)^{c}$ \\
\hline HDL-c (mmol) & $1,24(0,27)^{b, c}$ & $1,10(0,27)$ & $1,02(0,27)^{b}$ & $1,03(0,31)^{c}$ \\
\hline TG (mmol) & $0,55(0,54)^{a, b, c}$ & $0,86(0,99)^{a}$ & $0,99(0,59)^{b}$ & $1,10(0,56)^{c}$ \\
\hline VAl & $0,519(0,551)^{\mathrm{a}, \mathrm{b}, \mathrm{c}}$ & $0,965(1,012)^{a}$ & $1,065(928)^{b}$ & $1,322(0,800)^{c}$ \\
\hline \multicolumn{5}{|c|}{ Feminino } \\
\hline \multirow[t]{2}{*}{ Variáveis } & Eutrófico & Sobrepeso & Obesidade & $\begin{array}{c}\text { Obesidade } \\
\text { grave }\end{array}$ \\
\hline & $(n=41)$ & $(n=67)$ & $(n=40)$ & $(n=23)$ \\
\hline Idade (anos) & $15(3)$ & $16(2)$ & $16(1)$ & $16(2)$ \\
\hline $\mathrm{CC}(\mathrm{cm})$ & $68,86 \pm 6,35^{a, b, c}$ & $82,39 \pm 7,21^{a, d, e}$ & $87,83 \pm 6,22^{b, d, f}$ & $96,02 \pm 14,69$ ce, $f$ \\
\hline HDL-c (mg) & $53,9(15,00)^{a}$ & $47,60(17,30)^{\mathrm{a}}$ & $44,80(13,15)$ & $47,80(15,90)$ \\
\hline TG (mg) & $59,00(48,05)^{a, b, c}$ & $82,00(74)^{a}$ & $81,50(53,00)^{b}$ & $98,00(88,90)^{c}$ \\
\hline HDL-c (mmol) & $1,40(0,39)^{\mathrm{a}}$ & $1,23(0,45)^{a}$ & $1,16(0,34)$ & $1,24(0,41)$ \\
\hline TG (mmol) & $0,667(0,54)^{a, b, c}$ & $0,92(0,84)^{a}$ & $0,92(0,60)^{b}$ & $1,10(1,00)^{c}$ \\
\hline VAl & $0,707(0,823)^{a, b, c}$ & $1,351(1,138)^{\mathrm{a}}$ & $1,354(1,381)$ & $1,400(1,249)^{c}$ \\
\hline
\end{tabular}

Significância adotada $\mathrm{p} \leq 0,05$. Anova oneway para medidas paramétricas: Kruskal-Wallis para medidas não paramétricas; $a=$ diferença entre eutrófico e sobrepeso; $b=$ diferença entre eutrófico e obesidade; $c$ = diferença entre eutrófico e obesidade grave; $d=$ diferença entre sobrepeso e obesidade; $\mathrm{e}=$ diferença entre sobrepeso $\mathrm{e}$ obesidade grave; $f$ = diferença entre obesidade e obesidade grave.

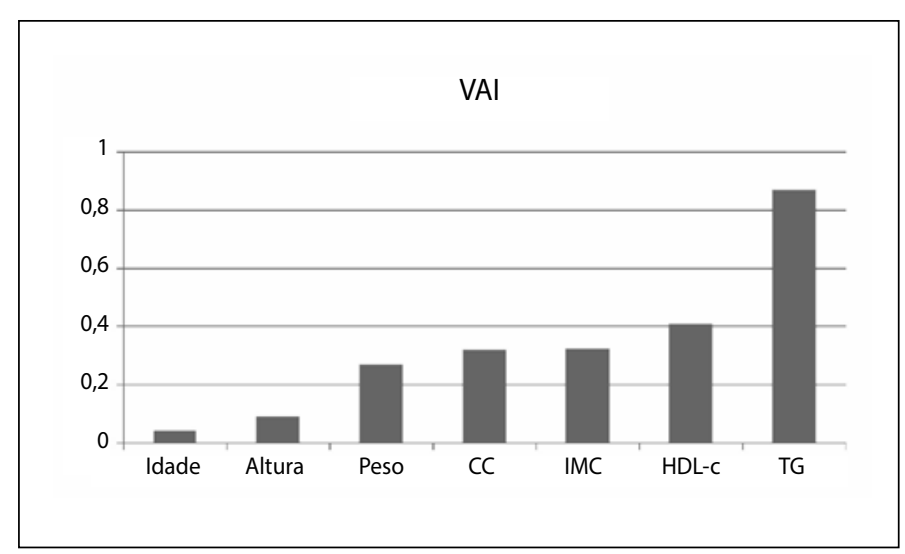

Figura 1. Coeficiente de Correlação de Pearson entre o VAl e os demais parâmetros analisados (o resultado da correlação do VAI vs HDL-c foi invertido para fins de comparação).
Tabela 4. Distribuição de frequência absoluta e relativa de acordo com alterações de IMC, CC, TG, HDL-c e VAl separados por sexo.

\begin{tabular}{c|c|c|c|c|c|c}
\hline \multirow{2}{*}{ Variáveis } & \multicolumn{2}{|c|}{ Masculino } & \multicolumn{2}{c|}{ Feminino } & \multicolumn{2}{c}{ Total } \\
\cline { 2 - 7 } & Normal & Alterado & Normal & Alterado & Normal & Alterado \\
\hline $\mathrm{IMC}\left(\mathrm{kg} / \mathrm{m}^{2}\right)$ & $26(21,8 \%)$ & $93(78,2 \%)$ & $41(24 \%)$ & $130(76 \%)$ & $67(23,1 \%)$ & $223(76,9 \%)$ \\
\hline $\mathrm{CC}(\mathrm{cm})$ & $66(55,5 \%)$ & $53(44,5 \%)$ & $137(80,1 \%)$ & $34(19,9 \%)$ & $203(70 \%)$ & $87(30 \%)$ \\
\hline $\mathrm{TG}(\mathrm{mg} / \mathrm{dL})$ & $108(90,8 \%)$ & $11(9,2 \%)$ & $153(89,5 \%)$ & $18(10,5 \%)$ & $261(90 \%)$ & $29(10 \%)$ \\
\hline $\mathrm{HDL}-\mathrm{C}(\mathrm{mg} / \mathrm{dL})$ & $62(52,1 \%)$ & $57(47,9 \%)$ & $110(64,3 \%)$ & $61(35,7 \%)$ & $172(59,3 \%)$ & $118(40,7)$ \\
\hline $\mathrm{VAl}$ & $113(95 \%)$ & $6(5 \%)$ & $151(88,3 \%)$ & $20(11,7 \%)$ & $264(91 \%)$ & $26(9 \%)$ \\
\hline
\end{tabular}

\section{DISCUSSÃO}

Al-Daghri et al. ${ }^{20}$ em um estudo com crianças e adolescentes árabes, encontraram valores mais elevados para CC, VAl e TG no sexo feminino e para HDL-c no sexo masculino. Já em um estudo com adultos, Ahmad e Haddad ${ }^{21}$ encontraram maiores valores em todas as variáveis no sexo feminino. No presente estudo os resultados para a variável CC divergiu de ambos os estudos referidos acima, e a variável HDL-c apenas em relação aos achados de Al-Daghri et al. ${ }^{20}$.

Os níveis de HDL-c apresentam uma tendência à redução na adolescência em ambos os sexos, porém mais acentuada em meninos e os hormônios sexuais femininos atuam como fator de proteção ao perfil lipídico em meninas púberes, aumentando HDL-c e diminuindo LDL-c $c^{22}$. Esta situação se assemelha aos resultados encontrados de HDL-c maiores para o sexo feminino (Tabela 1).

Garcés et al..$^{23}$, estudando uma proposta de VAl para a população pediátrica, encontraram um aumento da medida de CC conforme o estado nutricional, porém, constataram que o VAl e o TG também tiveram seus valores aumentados de acordo com o IMC. Eles verificaram que, com incrementos do estado nutricional, os parâmetros antropométricos e metabólicos (IMC, CC, HDL-c eTG) também pioraram em ambos os sexos.

O VAl foi correlacionado com a CC e o IMC (Figura 1), assemelhando-se parcialmente aos achados de Thivel et al. ${ }^{24}$ que constataram uma correlação significativa entre o VAl e a CC em adolescentes obesos, mas não entre o VAl e o IMC.

Os resultados encontrados demonstraram uma tendência, independente do sexo, para o desenvolvimento de fatores de risco cardiometabólicos à medida que a CC aumenta, em conformidade com os achados de Knowles et al. ${ }^{25}$

Martínez-Gómez et al. ${ }^{26}$ e Pavão et al. ${ }^{27}$ encontraram associação semelhante entre CC e dislipidemia. É importante destacar que a obesidade central pode ser um importante fator para valores alterados no perfil lipídico e aumento do risco cardiometabólico em adolescentes ${ }^{26,27}$, tornando o VAl um instrumento diagnóstico relevante para avaliação clínica.

O acúmulo de gordura corporal é um dos principais fatores de risco de doenças cardiometabólicas ${ }^{28}$, o que condiz com o aumento do risco observado nos participantes com excesso de peso (2,3x) e CC alterada (1,5x).

O perfil de TG tem se mostrado um importante preditor do risco cardiometabólico por conta de suas associações com as funções hemodinâmicas e cardiovasculares ${ }^{29}$. Foi observado uma forte correlação entre o VAl e o TG dos adolescentes investigados, situação corroborada por vários autores em diferentes populações ${ }^{13,30}$, o que reforça a utilização do VAl na predição de risco cardiometabólico.

Para Amato et al. ${ }^{19}$, em consequência do aumento de peso, o armazenamento excessivo de TG em adipócitos induz a alterações como a resistência insulínica, sendo uma das características da disfunção do tecido adiposo. Os mesmos destacam a importância da utilização do VAl para a avaliação do risco cardiometabólico.

Al-Daghri et al..$^{20}$ encontraram relação direta do VAl com as secreções do tecido adiposo, sendo um medidor indireto dos níveis de adiponectina e dos distúrbios do metabolismo da glicose, enfatizando assim a 
utilização do VAl como ferramenta simples para a marcação de risco cardiometabólico em concordancia com Schuster et al. ${ }^{13}$.

É importante ressaltar que os preditores utilizados por Al-Daghri et al. ${ }^{20}$ e Garcés et al. ${ }^{23}$ são novas propostas de VAl, com base no modelo original de Amato et. al. ${ }^{7}$, adaptados para a população pediátrica em modelo específico para cada sexo. A limitação encontrada na comparação entre os métodos de predição e a falta do estabelecimento de um critério no modelo proposto por Garcés et al. ${ }^{23}$, não enfraquecem a similaridade dos achados de ambos os estudos, pois os modelos de predição seguem a mesma base do modelo original.

Uma limitação encontrada no presente estudo foi a determinação do estágio maturacional dos adolescentes, uma vez que, o perfil lipídico varia conforme a fase de desenvolvimento maturacional. Além disso, o desenho transversal não permitiu avaliar a relação temporal entre as medidas indicadoras de adiposidade e componentes do VAl, porém permitiu identificar a influência do estado nutricional e o risco cardiometabólico.

\section{CONCLUSÕES}

O estado nutricional é capaz de influenciar as variáveis de risco cardiometabólico, VAl, CC, TG e HDL-c, em crianças e adolescentes. Nesta população, independente do sexo, à medida que o valor do IMC se eleva, há progressão de alterações metabólicas. Além disso, alterações do estado nutricional e da circunferência de cintura aumentam a chance de risco cardiometabólico. Em complemento, o VAl apresentou uma correlação significativa com o TG, HDL-C, IMC e CC das crianças e adolescentes do estudo, podendo assim ser visto como alternativa de predição do risco cardiometabólico. Não obstante, é importante ressaltar a necessidade de trabalhos que comparem a eficácia do VAl e sua aplicabilidade na população pediátrica.

\section{AGRADECIMENTOS}

Os autores agradecem ao CNPq, Ministério da Saúde, Fundação Araucária, Universidade Estadual de Maringá, Hospital Universitário de Maringá, Prefeitura Municipal e escolas públicas de Formosa do Oeste pelo apoio dado a esta pesquisa. Agradecimento especial a Carlos Andrés Lopera Barrero, pela tradução do resumo para o espanhol e a todos os participantes do Núcleo de Estudos Multiprofissional da Obesidade (NEMO) no auxílio da coleta dos dados.

Todos os autores declararam não haver qualquer potencial conflito de interesses referente a este artigo.

CONTRIBUIÇÕES DOS AUTORES: Cada autor contribuiu individual e significativamente para o desenvolvimento do manuscrito. RPO (0000-0002-3709-4579)* e SPBL (0000-0003-1583-0317)*, chefiaram e coletaram os dados em campo, JMR (0000-0002-3003-1786)*, TLCS (0000-0002-6472-4206)*, JCL (0000-0002-6843-4150)* e SPBL, realizaram a pesquisa bibliográfica. RPO, JMR, ARM (0000-0002-5558-9188)* participaram de redação e revisão crítica do manuscrito. JMR, ARM, RPD (0000-0002-7630345x)*, AAM (0000-0002-7665-2000)*, TLCS, JCL e CMOT (0000-0003-4220-5533)*, coletaram dados em campo. JMR, ARM e AAM e RPO, contribuíram na discussão dos resultados. RPD, NNJ (0000-0002-6862-7868)* e RPO, realizaram a análise estatística dos dados. RPD, AAM, TLCS e CMOT, contribuíram com a redação do manuscrito. NNJ, concebeu o estudo, coordenou o grupo de pesquisa e realizou a revisão crítica do manuscrito. ARM, contribuiu com o conceito intelectual do estudo. RPO, JMR e NNJ, contribuíram com o conceito intelectual do estudo e aprovação final da versão do manuscrito. *ORCID (Open Researcher and Contributor ID).

\section{REFERÊNCIAS}

1. Brasil. Ministério da Saúde. Cadernos de atenção básica: estratégias para o cuidado da pessoa com doença crônica obesidade. Braślia, DF: Ministério da Saúde; 2014.

2. World Health Organization (WHO). Commission on ending childhood obesity [acessoem 2016 nov 17]. Disponivel em : http://www.who.int/end- childhood-obesity/en/.

3. Ng M, Fleming T, Robinson M, Thomson B, Graetz N, Margono C, et al. Global, regional, and national prevalence of overweight and obesity in children and adults during 1980-2013: a systematic analysis for the Global Burden of Disease Study 2013. Lancet. 2014;384(9945):766-81.

4. Brasil. Ministério da Saúde, Secretaria de Vigilância em Saúde. VIGITEL 2014: vigilância de fatores de risco e proteção para doenças crônicas por inquérito telefônico. Braślia, DF: Ministério da Saúde; 2015.

5. Rezende FAC, Lima LEF, Castro SCF, Rosado GP, Ribeiro RCL. Aplicabilidade do índice de massa corporal na avaliação da gordura corporal. Rev Bras Med Esporte. 2010;16:90-4.

6. Tanamas SK, Permatahati V, Ng WL, Backholer K, Wolfe R, Shaw JE, et al. Estimating the proportion of metabolic health outcomes attributable to obesity: a cross-sectional exploration of body mass index and waist circumference combinations. BMC Obes. 2016;3:4

7. Amato MC, Giordano C, Galia M, Criscimanna A, Vitabile S, Midiri M, et al. Visceral Adiposity Index: a reliable indicator of visceral fat function associated with cardiometabolic risk. Diabetes Care. 2010;33(4):920-2.

8. Hayashi T, Boyko EJ, Leonetti DL, McNeely MJ, Newell-Morris L, Kahn SE, et al. Visceral adiposity and the risk of impaired glucose tolerance: a prospective study among Japanese Americans. Diabetes Care. 2003;26(3):650-5

9. Petribú M de MV, Guimarães FJ de SP, Cabral PC, Santos EMC, Diniz A da S, de Arruda IKG. Desenvolvimento e validação de equação preditiva da gordura visceral em mulheres jovens. Rev Bras Cineantropom Desempenho Hum. 2012;14(3):333-42.

10. Al-Daghri NM, Al-Attas OS, Wani K, Alnaami AM, Sabico S, Al-Ajlan A, et al. Sensitivity of various adiposity indices in identifying cardiometabolic diseases in Arab adults. Cardiovasc Diabetol. 2015;14:101.

11. Han L, Fu KL, Zhao J, Wang ZH, Tang MX, Wang J, et al.Visceral adiposity index score indicated the severity of coronary heart disease in Chinese adults. Diabetol Metab Syndr. 2014;6(1):143.

12. Ding Y, Gu D, Zhang Y, Han W, Liu H, Qu Q. Significantly increased visceral adiposity index in prehypertension. PLoSOne. 2015;10(4):e0123414.

13. Schuster J, Vogel P, Eckhardt C, Morelo SD. Applicability of the visceral adiposity index (VAI) in predicting components of metabolic syndrome in young adults. Nutr Hosp. 2014;30(4):806-12.

14. Zen V. Doença arterial coronariana: avaliação através de marcadores não convencionais: adiponectina de alto peso molecular, VAl e LAP [dissertação]. Rio Grande do Sul, RS: Universidade Federal do Rio Grande do Sul; 2015.

15. Du T, Yu X, Zhang J, Sun X. Lipid accumulation product and visceral adiposity index are effective markers for identifying the metabolically obese normal-weight phenotype. Acta Diabetol. 2015;52(5):855-63.
16. Lohman TG, Roche AF, Martorell R, eds. Anthropometric standardization reference manual. Champaign IL: Human Kinetics Books; 1988.

17. Cole TJ, Lobstein T. Extended international (IOTF) body mass index cut-offs for thinness, overweight and obesity. PediatrObes. 2012;7(4):284-94.

18. Amato MC, Giordano C, Pitrone M, Galluzzo A. Cut-off points of the visceral adiposity index (VAI) identifying a visceral adipose dysfunction associated with cardiometabolic risk in a Caucasian Sicilian population. Lipids Health Dis. 2011;10:183.

19. Amato MC, Giordano C. Visceral adiposity index: an indicator of adipose tissue dysfunction. Int $J$ Endocrinol. 2014;2014:730827.

20. Al-Daghri NM, Al-Attas OS, Alokail M, Alkharfy K, Wani K, Amer OE, et al. Does visceral adiposity index signify early metabolic risk in children and adolescents?: association with insulin resistance, adipokines, and subclinical inflammation. Pediatr Res. 2014;75(3):459-63.

21. Numan Ahmad M, Halim Haddad F. Suitability of visceral adiposity index as a marker for cardiometabolic risks in jordanian adults. Nutr Hosp. 2015;32(6):2701-9.

22. Rabelo LM. Atherosclerotic risk factors in adolescence. J Pediatr (Rio J). 2001;77(Suppl 2):S153-64

23. Garcés MJ, Hernández J, Queipo G, Klünder-klünder M, Bustos M, Herrera A, et al. Novel gender-specific visceral adiposity index for Mexican pediatric population. Rev Med del Hosp Gen México; 2014;77(4):153-9.

24. Thivel D, O'Malley G, Pereira B, Duché P, Aucouturier J. Comparison of total body and abdominal adiposity indexes to dual x-ray absorptiometry scan in obese adolescents. Am J Hum Biol. 2015;27(3):334-8.

25. Knowles KM, Paiva LL, Sanchez SE, Revilla L, Lopez T, Yasuda MB, et al. Waist Circumference, Body Mass Index, and Other Measures of Adiposity in Predicting Cardiovascular Disease Risk Factors among Peruvian Adults. Int J Hypertens. 2011;2011:931402

26. Martínez-Gómez D, Eisenmann JC, Gómez-Martínez S, Veses A, Marcos A, Veiga OL. Sedentary behavior, adiposity and cardiovascular risk factors in adolescents. The AFINOS study. Rev Esp Cardiol. 2010;63(3):277-85

27. Pavão FH, Schiavoni D, Pizzi J, Silva KE de S, Junior HS. Dislipidemia em adolescentes residentes em um município do Paraná e sua associação com a obesidade abdominal. Rev da Educ Física. 2015;26(3):473-81.

28. Miranda VPN, Amorim PR dos S, Oliveira NCB, Peluzio M do CG, Priore SE. Effect of physical activity on cardiometabolic markers in adolescents : systematic review. Rev Bras Med Esporte. 2016;22(3):235-42.

29. Grundy SM, Cleeman JI, Daniels SR, Donato KA, Eckel RH, Franklin BA, et al. Diagnosis and management of the metabolic syndrome: an American Heart Association/National Heart, Lung, and Blood Institute scientific statement: Executive Summary. Crit Pathw Cardiol. 2005;4(4):198-203.

30. Goldani H, Adami FS, Antunes MT, Rosa LH, Fassina P, Quevedo Grave MT, et al. Applicatility of the viscera adiposity index (vai) in the prediction of the components of the metabolic syndrome in elderly. Nutr Hosp. 2015;32(4):1609-15. 